Homology, Homotopy and Applications, vol. 16(1), 2014, pp.65-81

\title{
FREE 2-RANK OF SYMMETRY OF PRODUCTS OF MILNOR MANIFOLDS
}

\author{
MAHENDER SINGH
}

(communicated by Donald M. Davis)

\begin{abstract}
A real Milnor manifold is the non-singular hypersurface of degree $(1,1)$ in the product of two real projective spaces. These manifolds were introduced by Milnor to give generators for the unoriented cobordism algebra, and they admit free actions by elementary abelian 2-groups. In this paper, we obtain some results on the free 2-rank of symmetry of products of finitely many real Milnor manifolds under the assumption that the induced action on mod 2 cohomology is trivial. Similar results are obtained for complex Milnor manifolds that are defined analogously. Here the free 2-rank of symmetry of a topological space is the maximal rank of an elementary abelian 2-group that acts freely on that space.
\end{abstract}

\section{Introduction}

One of the basic problems in the theory of transformation groups is to determine the structure of a group that acts in a specific way on a given topological space. The problem of determining finite groups that can act freely on spheres has been of special interest. A classical result of Smith [29] says that if a group acts freely on a sphere, then all its abelian subgroups are cyclic. Conversely, Swan $[\mathbf{3 0}]$ proved that any group satisfying this condition acts freely on a finite complex with the homotopy type of a sphere. Notice that a finite abelian group is cyclic if and only if it does not contain a subgroup of the form $\mathbb{Z} / p \oplus \mathbb{Z} / p$ for any prime $p$. Thus, $\mathbb{Z} / p \oplus \mathbb{Z} / p$ cannot act freely on a sphere. However, $\mathbb{Z} / p$ does act freely on a sphere, where the sphere must be odd dimensional for odd $p$. These results motivated the concept of free $p$-rank of symmetry of a topological space $X$ for a prime $p$, introduced in [1], and defined as

$$
\operatorname{frk}_{p}(X)=\max \left\{r \mid(\mathbb{Z} / p)^{r} \text { acts freely on } X\right\} .
$$

Determining the free $p$-rank of symmetry of a topological space is an interesting problem and has been considered for many spaces. In view of the Smith theory, we

Received August 9, 2013; published on March 12, 2014.

2010 Mathematics Subject Classification: Primary 57S25; Secondary 57S17, 55T10.

Key words and phrases: free rank, Milnor manifold, Leray-Serre spectral sequence, Steenrod algebra. Article available at http://dx.doi.org/10.4310/HHA.2014.v16.n1.a4

Copyright (C) 2014, International Press. Permission to copy for private use granted. 
have

$$
\operatorname{frk}_{p}\left(\mathbb{S}^{n}\right)= \begin{cases}1 & \text { if } n \text { is odd and } p \text { is arbitrary } \\ 1 & \text { if } n \text { is even and } p=2 \\ 0 & \text { if } n \text { is even and } p>2 .\end{cases}
$$

The problem of extending this result to products of finitely many spheres has been of great interest to many topologists. Oliver [28] proved that every finite group acts freely on a product of spheres. For a finite group $G$, define

$$
\begin{gathered}
h(G)=\min \left\{s \mid G \text { acts freely on } \mathbb{S}^{n_{1}} \times \cdots \times \mathbb{S}^{n_{s}}\right\} \text { and } \\
r(G)=\max \left\{t \mid(\mathbb{Z} / p)^{t} \leqslant G \text { for some prime } p\right\} .
\end{gathered}
$$

Attempts to extend Smith's result to products of finitely many spheres led to the following conjecture by Benson and Carlson [6].

Conjecture I. $h(G)=r(G)$ for each finite group $G$.

Conjecture I implies the following well-known conjecture $[\mathbf{1}, \mathbf{2}, \mathbf{9}]$ regarding free actions of elementary abelian $p$-groups on products of spheres.

Conjecture II. $\operatorname{frk}_{p}\left(\mathbb{S}^{2 n_{1}+1} \times \cdots \times \mathbb{S}^{2 n_{k}+1}\right)=k$ for each prime $p$ and integer $k \geqslant 0$.

For a single sphere it is simply the result of Smith [29]. For products of two spheres it was already proved by Conner [11], and for products of three spheres it was proved to be true by Heller [20]. Carlsson $[\mathbf{9}, \mathbf{1 0}]$ proved the conjecture for products of equidimensional spheres under the assumption of trivial induced action on cohomology. Adem and Browder [1] proved that $\operatorname{frk}_{p}\left(\left(\mathbb{S}^{n}\right)^{k}\right)=k$ with the only remaining cases as $p=2$ and $n=1,3,7$. Some time later, Yalçın [32] proved that $\operatorname{frk}_{2}\left(\left(\mathbb{S}^{1}\right)^{k}\right)=k$. For an integer $n$, let

$$
\eta(n)= \begin{cases}0 & \text { if } n \text { is even } \\ 1 & \text { if } n \text { is odd }\end{cases}
$$

Then the most comprehensive result is due to Hanke [19], who proved that if $p>$ $3\left(n_{1}+\cdots+n_{k}\right)$, then

$$
\operatorname{frk}_{p}\left(\mathbb{S}^{n_{1}} \times \cdots \times \mathbb{S}^{n_{k}}\right)=\eta\left(n_{1}\right)+\cdots+\eta\left(n_{k}\right) .
$$

In a very recent work [27], Okutan and Yalçın proved Conjecture II in the case where the dimensions $\left\{n_{i}\right\}$ are high compared to all the differences $\left|n_{i}-n_{j}\right|$ between the dimensions.

Recall that $\mathbb{Z} / 2$ is the only finite group that can act freely on an even-dimensional sphere. This result was extended to products of finitely many even-dimensional spheres by Cusick $[\mathbf{1 4}, \mathbf{1 5}]$. He proved that if $G$ is a finite group acting freely on $\mathbb{S}^{2 n_{1}} \times \cdots \times$ $\mathbb{S}^{2 n_{k}}$ with trivial induced action on $\bmod 2$ cohomology, then $G \cong(\mathbb{Z} / 2)^{r}$ for some $r \leqslant k$. In particular, when the induced action on mod 2 cohomology is trivial, then $\operatorname{frk}_{2}\left(\mathbb{S}^{2 n_{1}} \times \cdots \times \mathbb{S}^{2 n_{k}}\right)=k$.

Although a lot of work has been done for products of spheres, free $p$-rank of symmetry of many other interesting spaces is still not known. An immediate extension of the problem from spheres and their products is to consider spherical space forms and their products. Let $p$ be an odd prime. A lens space $L_{p}^{2 n-1}$ is an odd-dimensional 
spherical space form obtained as the quotient of the standard $\mathbb{Z} / p$ action on $\mathbb{S}^{2 n-1}$. Allday [5] conjectured that

$$
\operatorname{frk}_{p}\left(L_{p}^{2 n_{1}-1} \times \cdots \times L_{p}^{2 n_{k}-1}\right)=k .
$$

The equidimensional case of the conjecture was proved by Yalçın [31]. The general case of the conjecture seems still open.

The problem of computing the free 2-rank of symmetry of products of projective spaces was considered by Cusick. It is known that $\mathbb{C} P^{n}$ admits a free action by a finite group if and only if $n$ is odd, in which case the only possible group is $\mathbb{Z} / 2$. Cusick $[\mathbf{1 6}]$ proved that

$$
\operatorname{frk}_{2}\left(\mathbb{C} P^{n_{1}} \times \cdots \times \mathbb{C} P^{n_{k}}\right)=\eta\left(n_{1}\right)+\cdots+\eta\left(n_{k}\right) .
$$

For an integer $n$, let

$$
\theta(n)= \begin{cases}0 & \text { if } n \text { is even } \\ 1 & \text { if } n \equiv 1 \bmod 4 \\ 2 & \text { if } n \equiv 3 \bmod 4\end{cases}
$$

Cusick [13] investigated the real case and conjectured that if the induced action on mod 2 cohomology is trivial, then

$$
\operatorname{frk}_{2}\left(\mathbb{R} P^{n_{1}} \times \cdots \times \mathbb{R} P^{n_{k}}\right)=\theta\left(n_{1}\right)+\cdots+\theta\left(n_{k}\right) .
$$

He proved the conjecture when $n_{i} \not \equiv 3 \bmod 4$ for each $1 \leqslant i \leqslant k$. Adem and Yalçın [3] proved the conjecture for products of equidimensional real projective spaces without the assumption of trivial induced action on mod 2 cohomology. Later, Yalçın [32] proved the conjecture when $n_{i}$ is odd for each $1 \leqslant i \leqslant k$. The general case of the conjecture is still open.

A product of two projective spaces can be considered as the total space of a trivial projective space bundle over a projective space. It is an interesting question to determine the free rank of symmetry of the total space of a twisted projective space bundle over a projective space. Milnor manifolds are fundamental examples of such spaces. These manifolds were introduced by Milnor [25] in search for generators for the unoriented cobordism algebra, and are non-singular hypersurfaces of degree $(1,1)$ in the product of two projective spaces (see Section 2 for detailed definitions). The purpose of this paper is to obtain some results regarding the free 2-rank of symmetry of products of finitely many Milnor manifolds. We will consider both real and complex Milnor manifolds. We will prove our results by adopting Cusick's method, which depends on some results of Carlsson.

Let $\mathbb{R} H_{r, s}$ and $\mathbb{C} H_{r, s}$ denote the real and the complex Milnor manifold, respectively (see Section 2 for notation). Let $X \simeq_{2} Y$ mean that $X$ and $Y$ are topological spaces having isomorphic mod 2 cohomology algebra. Then the main results are as follows.

Theorem 1.1. Let $(\mathbb{Z} / 2)^{r}$ act freely on a finite-dimensional $C W$-complex $X \simeq_{2} \prod_{i=1}^{n} \mathbb{R} H_{r_{i}, s_{i}}$ with $1 \leqslant s_{i} \leqslant r_{i}$ for each $1 \leqslant i \leqslant n$. Suppose that the induced action on mod 2 cohomology of $X$ is trivial. Then

1. $r \leqslant 2\left(\eta\left(s_{1}\right)+\eta\left(r_{1}\right)+\cdots+\eta\left(s_{n}\right)+\eta\left(r_{n}\right)\right)$,

2. $r \leqslant\left(\eta\left(s_{1}\right)+\eta\left(r_{1}\right)+\cdots+\eta\left(s_{n}\right)+\eta\left(r_{n}\right)\right)$ if $s_{i}, r_{i} \not \equiv 3 \bmod 4$ for each $1 \leqslant i \leqslant n$. 
Theorem 1.2. Let $(\mathbb{Z} / 2)^{r}$ act freely on a finite-dimensional $C W$-complex $X \simeq_{2} \prod_{i=1}^{n} \mathbb{C} H_{r_{i}, s_{i}}$ with $1 \leqslant s_{i} \leqslant r_{i}$ for each $1 \leqslant i \leqslant n$. Suppose that the induced action on mod 2 cohomology of $X$ is trivial. Then

1. $r \leqslant 3\left(\eta\left(s_{1}\right)+\eta\left(r_{1}\right)+\cdots+\eta\left(s_{n}\right)+\eta\left(r_{n}\right)\right)$,

2. $r \leqslant\left(\eta\left(s_{1}\right)+\eta\left(r_{1}\right)+\cdots+\eta\left(s_{n}\right)+\eta\left(r_{n}\right)\right)$ if $s_{i}, r_{i} \not \equiv 3 \bmod 4$ for each $1 \leqslant i \leqslant n$.

\section{Definition and cohomology of Milnor manifolds}

Milnor manifolds were introduced by Milnor [25] in search for generators for the unoriented cobordism algebra. Let $r$ and $s$ be integers such that $0 \leqslant s \leqslant r$. A real Milnor manifold, denoted by $\mathbb{R} H_{r, s}$, is the non-singular hypersurface of degree $(1,1)$ in the product $\mathbb{R} P^{r} \times \mathbb{R} P^{s}$. It is a $(s+r-1)$-dimensional closed smooth manifold and can also be described in terms of homogeneous coordinates of real projective spaces as

$$
\mathbb{R} H_{r, s}=\left\{\left(\left[x_{0}, \ldots, x_{r}\right],\left[y_{0}, \ldots, y_{s}\right]\right) \in \mathbb{R} P^{r} \times \mathbb{R} P^{s} \mid x_{0} y_{0}+\cdots+x_{s} y_{s}=0\right\} .
$$

Equivalently, a real Milnor manifold can be defined as the total space of the fiber bundle

$$
\mathbb{R} P^{r-1} \stackrel{i}{\hookrightarrow} \mathbb{R} H_{r, s} \stackrel{\pi}{\longrightarrow} \mathbb{R} P^{s} .
$$

This is actually the projectivization of the vector bundle

$$
\mathbb{R}^{r} \hookrightarrow E^{\perp} \longrightarrow \mathbb{R} P^{s},
$$

which is the orthogonal complement in $\mathbb{R} P^{s} \times \mathbb{R}^{r+1}$ of the canonical line bundle

$$
\mathbb{R} \hookrightarrow E \longrightarrow \mathbb{R} P^{s},
$$

where $E=\left\{([x], y) \in \mathbb{R} P^{s} \times \mathbb{R}^{r+1} \mid y \in[x]\right\}$.

These manifolds are important as the unoriented cobordism algebra of smooth manifolds is generated by the cobordism classes of $\mathbb{R} P^{k}$ and $\mathbb{R} H_{r, s}[\mathbf{2 5}$, Lemma 1]. Therefore, determining their various invariants is an important problem.

In a similar way, a complex Milnor manifold, denoted by $\mathbb{C} H_{r, s}$, is the nonsingular hypersurface of degree $(1,1)$ in the product $\mathbb{C} P^{r} \times \mathbb{C} P^{s}$. It is a $2(s+r-1)$ dimensional closed smooth manifold and can also be described in terms of homogeneous coordinates as

$$
\mathbb{C} H_{r, s}=\left\{\left(\left[z_{0}, \ldots, z_{r}\right],\left[w_{0}, \ldots, w_{s}\right]\right) \in \mathbb{C} P^{r} \times \mathbb{C} P^{s} \mid z_{0} \bar{w}_{0}+\cdots+z_{s} \bar{w}_{s}=0\right\} .
$$

Equivalently, as in the real case, a complex Milnor manifold can be defined as the total space of the fiber bundle

$$
\mathbb{C} P^{r-1} \stackrel{i}{\hookrightarrow} \mathbb{C} H_{r, s} \stackrel{\pi}{\longrightarrow} \mathbb{C} P^{s} .
$$

It is known due to Conner and Floyd $\left[\mathbf{1 2}\right.$, p. 63] that $\mathbb{C} H_{r, s}$ is unoriented cobordant to $\mathbb{R} H_{r, s} \times \mathbb{R} H_{r, s}$.

These manifolds have been well studied in the past. See, for example, $[\mathbf{1 7}, \mathbf{2 1}, \mathbf{2 6}$ for recent results. Their cohomology algebra is also well known, and we will use it in the proofs of our main theorems. 
Lemma $2.1([\mathbf{8}, \mathbf{2 6}])$. Let $0 \leqslant s \leqslant r$. Then the $\bmod 2$ cohomology algebra of a Milnor manifold is given as follows:

1. $H^{*}\left(\mathbb{R} H_{r, s} ; \mathbb{Z} / 2\right) \cong \mathbb{Z} / 2[a, b] /\left\langle a^{s+1}, b^{r}+a b^{r-1}+\cdots+a^{s} b^{r-s}\right\rangle$, where $a$ and $b$ are homogeneous elements of degree 1 each.

2. $H^{*}\left(\mathbb{C} H_{r, s} ; \mathbb{Z} / 2\right) \cong \mathbb{Z} / 2[g, h] /\left\langle g^{s+1}, h^{r}+g h^{r-1}+\cdots+g^{s} h^{r-s}\right\rangle$, where $g$ and $h$ are homogeneous elements of degree 2 each.

Note that $\mathbb{R} H_{r, 0}=\mathbb{R} P^{r-1}$ and $\mathbb{C} H_{r, 0}=\mathbb{C} P^{r-1}$. Since the free 2-rank of symmetry of products of projective spaces has already been considered by Cusick [13], we henceforth assume that $1 \leqslant s \leqslant r$.

\section{Examples of free actions of elementary abelian 2-groups}

Just like projective spaces, Milnor manifolds also admit free actions by elementary abelian 2-groups.

\subsection{The real case}

First, we construct free actions on $\mathbb{R} H_{r, s}$ for various values of $s$ and $r$.

3.1.1. When $s=r$

The involution on $\mathbb{R} P^{s} \times \mathbb{R} P^{s}$ given by

$$
\left(\left[x_{0}, \ldots, x_{s}\right],\left[y_{0}, \ldots, y_{s}\right]\right) \longmapsto\left(\left[y_{0}, \ldots, y_{s}\right],\left[x_{0}, \ldots, x_{s}\right]\right)
$$

restricts to a free involution $A: \mathbb{R} H_{s, s} \longrightarrow \mathbb{R} H_{s, s}$.

3.1.2. When $s, r \equiv 1 \bmod 4$

Let $n=s, r$ and $S_{1}: \mathbb{R} P^{n} \longrightarrow \mathbb{R} P^{n}$ be the free involution given by

$$
\left[x_{0}, x_{1}, \ldots, x_{n-1}, x_{n}\right] \longmapsto\left[-x_{1}, x_{0}, \ldots,-x_{n}, x_{n-1}\right] .
$$

Then the restriction of $S_{1} \times S_{1}: \mathbb{R} P^{r} \times \mathbb{R} P^{s} \longrightarrow \mathbb{R} P^{r} \times \mathbb{R} P^{s}$ on $\mathbb{R} H_{r, s}$ gives a free involution

$$
A_{1}: \mathbb{R} H_{r, s} \longrightarrow \mathbb{R} H_{r, s}
$$

3.1.3. When $s, r \equiv 3 \bmod 4$

First, notice that the free involution $A_{1}$ is also defined in this case. Let $n=s, r$ and $S_{2}: \mathbb{R} P^{n} \longrightarrow \mathbb{R} P^{n}$ be the free involution given by

$$
\begin{aligned}
{\left[x_{0}, x_{1}, x_{2}, x_{3}, \ldots, x_{r-3}, x_{r-2}, x_{r-1}, x_{r}\right] } & \longmapsto\left[-x_{2}, x_{3}, x_{0},-x_{1}, \ldots,-x_{r-1}, x_{r}, x_{r-3},-x_{r-2}\right] .
\end{aligned}
$$

Then the restriction of $S_{2} \times S_{2}: \mathbb{R} P^{r} \times \mathbb{R} P^{s} \longrightarrow \mathbb{R} P^{r} \times \mathbb{R} P^{s}$ on $\mathbb{R} H_{r, s}$ gives a free involution

$$
A_{2}: \mathbb{R} H_{r, s} \longrightarrow \mathbb{R} H_{r, s} .
$$

Notice that $A_{1} \neq A_{2}, A_{1} A_{2}=A_{2} A_{1}$, and $A_{1} A_{2}$ acts freely on $\mathbb{R} H_{r, s}$. Therefore, $(\mathbb{Z} / 2)^{2}$ acts freely on $\mathbb{R} H_{r, s}$ when $s, r \equiv 3 \bmod 4$. 


\subsection{The complex case}

Now we construct free actions on $\mathbb{C} H_{r, s}$ for various values of $s$ and $r$.

3.2.1. When $s=r$

The involution on $\mathbb{C} P^{s} \times \mathbb{C} P^{s}$ given by

$$
\left(\left[z_{0}, \ldots, z_{s}\right],\left[w_{0}, \ldots, w_{s}\right]\right) \longmapsto\left(\left[w_{0}, \ldots, w_{s}\right],\left[z_{0}, \ldots, z_{s}\right]\right)
$$

restricts to a free involution $B: \mathbb{C} H_{s, s} \longrightarrow \mathbb{C} H_{s, s}$.

\subsubsection{When both $s$ and $r$ are odd}

It is known that $\mathbb{C} P^{n}$ admits a free action by a finite group if and only if $n$ is odd and in that case the only possible group is $\mathbb{Z} / 2$. Let $n=s, r$ and $T_{1}: \mathbb{C} P^{n} \longrightarrow \mathbb{C} P^{n}$ be the free involution given by

$$
\left[z_{0}, z_{1}, \ldots, z_{n-1}, z_{n}\right] \longmapsto\left[-\bar{z}_{1}, \bar{z}_{0}, \ldots,-\bar{z}_{n}, \bar{z}_{n-1}\right] .
$$

Then the restriction of $T_{1} \times T_{1}: \mathbb{C} P^{r} \times \mathbb{C} P^{s} \longrightarrow \mathbb{C} P^{r} \times \mathbb{C} P^{s}$ on $\mathbb{C} H_{r, s}$ gives a free involution

$$
B_{1}: \mathbb{C} H_{r, s} \longrightarrow \mathbb{C} H_{r, s}
$$

3.2.3. When $s$ is odd and $r$ is even

Let $T_{2}: \mathbb{C} P^{r} \longrightarrow \mathbb{C} P^{r}$ be the involution given by

$$
\left[z_{0}, z_{1}, \ldots, z_{r-2}, z_{r-1}, z_{r}\right] \longmapsto\left[-\bar{z}_{1}, \bar{z}_{0}, \ldots,-\bar{z}_{r-1}, \bar{z}_{r-2}, \iota z_{r}\right]
$$

where $\iota^{2}=-1$. Notice that $T_{2}$ is not a free involution. But the restriction of $T_{2} \times T_{1}$ : $\mathbb{C} P^{r} \times \mathbb{C} P^{s} \longrightarrow \mathbb{C} P^{r} \times \mathbb{C} P^{s}$ on $\mathbb{C} H_{r, s}$ gives a free involution

$$
B_{2}: \mathbb{C} H_{r, s} \longrightarrow \mathbb{C} H_{r, s} \text {. }
$$

\section{Preliminary results}

Here we recall some facts that we will use in this paper. We refer the reader to $[4,7,24]$ for details on the cohomology theory of transformation groups and spectral sequences. We refer to $[\mathbf{2 3}]$ for basic properties of Steenrod algebra. Throughout, we will use cohomology with $\mathbb{Z} / 2$ coefficients and will suppress it from cohomology notation. All spaces under consideration will be finite-dimensional CW-complexes. More generally, we can also consider paracompact spaces of finite cohomological dimension or finitistic spaces (which include paracompact spaces of finite covering dimension and compact Hausdorff spaces).

All group actions under consideration are assumed to be continuous. Let $G$ be a finite group acting on a space $X$, and let

$$
G \hookrightarrow E_{G} \longrightarrow B_{G}
$$

be the universal principal $G$-bundle. Let

$$
X_{G}=\left(X \times E_{G}\right) / G
$$

be the orbit space of the diagonal action on $X \times E_{G}$. Then the projection

$$
X \times E_{G} \longrightarrow E_{G}
$$


is $G$-equivariant and gives a fibration

$$
X \hookrightarrow X_{G} \longrightarrow B_{G}
$$

called the Borel fibration [7, Chapter IV]. Recall that for $G=(\mathbb{Z} / 2)^{r}$, we have

$$
B_{G}=\underbrace{\mathbb{R} P^{\infty} \times \cdots \times \mathbb{R} P^{\infty}}_{r \text { times }}
$$

and hence

$$
H^{*}\left(B_{G} ; \mathbb{Z} / 2\right)=\mathbb{Z} / 2\left[\alpha_{1}, \ldots, \alpha_{r}\right],
$$

where $\alpha_{i}$ is a homogeneous element of degree 1 for each $1 \leqslant i \leqslant r$.

We will exploit the Leray-Serre spectral sequence associated to a fibration as given by the following theorem.

Theorem 4.1 ([24], Theorem 5.2). Let $X \hookrightarrow E \longrightarrow B$ be a fibration. Then there is a first quadrant spectral sequence of algebras $\left\{E_{r}^{*, *}, d_{r}\right\}$, converging to $H^{*}(E)$ as an algebra, with

$$
E_{2}^{k, l}=H^{k}\left(B ; \mathcal{H}^{l}(X)\right),
$$

the cohomology of the base $B$ with local coefficients in the cohomology of the fiber $X$.

The product in $E_{r+1}^{*, *}$ is induced by the product in $E_{r}^{*, *}$ and the differentials are derivations. Further, there is an isomorphism of graded commutative algebra

$$
H^{*}(E) \cong \operatorname{Tot} E_{\infty}^{*, *}
$$

where $\operatorname{Tot} E_{\infty}^{* * *}$ is the total complex of $E_{\infty}^{*, *}$.

Theorem 4.2 ([24], Theorem 5.9). Let $X \stackrel{i}{\hookrightarrow} E \stackrel{\pi}{\longrightarrow} B$ be a fibration. Suppose that the system of local coefficients on $B$ is simple; then the edge homomorphisms

$$
\begin{gathered}
H^{k}(B)=E_{2}^{k, 0} \longrightarrow E_{3}^{k, 0} \longrightarrow \cdots \longrightarrow E_{k}^{k, 0} \longrightarrow E_{k+1}^{k, 0}=E_{\infty}^{k, 0} \subset H^{k}(E) \quad \text { and } \\
H^{l}(E) \longrightarrow E_{\infty}^{0, l}=E_{l+1}^{0, l} \subset E_{l}^{0, l} \subset \cdots \subset E_{2}^{0, l}=H^{l}(X)
\end{gathered}
$$

are the homomorphisms

$$
\pi^{*}: H^{k}(B) \longrightarrow H^{k}(E) \quad \text { and } i^{*}: H^{l}(E) \longrightarrow H^{l}(X) .
$$

Next we recall some results regarding elementary abelian 2-group actions on finitedimensional $\mathrm{CW}$-complexes.

Theorem 4.3 ([4], Theorem 3.10.4). Let $G=(\mathbb{Z} / 2)^{r}$ act freely on a finite-dimensional $C W$-complex $X$. Suppose that $\sum_{i \geqslant 0} r k\left(H^{i}(X)\right)<\infty$ and the induced action on $H^{*}(X)$ is trivial; then the Leray-Serre spectral sequence associated to $X \hookrightarrow X_{G} \longrightarrow$ $B_{G}$ does not degenerate at the $E_{2}$ term.

Proposition 4.4 ([4], Proposition 3.10.9 and Lemma 3.10.16). Let $G=(\mathbb{Z} / 2)^{r}$ act freely on a finite-dimensional $C W$-complex $X$. Then $H^{*}(X / G) \cong H^{*}\left(X_{G}\right)$. Further, if $H^{i}(X)=0$ for all $i>n$, then $H^{i}\left(X_{G}\right)=0$ for all $i>n$.

We will also use the following results regarding non-trivial common zeros of a system of homogeneous polynomials. 
Proposition 4.5 ([9], Proposition 1$)$. Let $G=(\mathbb{Z} / 2)^{r}$ and $f_{1}, \ldots, f_{n}$ be elements of $H^{m}\left(B_{G}\right)$ regarded as homogeneous polynomials of degree $m$ in $r$ variables. Then they have a non-trivial common zero in $(\mathbb{Z} / 2)^{r}$ if and only if there is a subgroup inclusion $j: \mathbb{Z} / 2 \hookrightarrow(\mathbb{Z} / 2)^{r}$ such that $j^{*}\left(f_{i}\right)=0$ for each $1 \leqslant i \leqslant n$, where $j^{*}: H^{*}\left(B_{G}\right) \rightarrow$ $H^{*}\left(B_{\mathbb{Z} / 2}\right)$ is the induced map on cohomology.

Proposition 4.6 ([9], Proposition 4$)$. Let $G=(\mathbb{Z} / 2)^{r}$ and $f_{1}, \ldots, f_{n}$ be elements of $H^{*}\left(B_{G}\right)$ regarded as homogeneous polynomials in $r$ variables. Suppose that the ideal $\left\langle f_{1}, \ldots, f_{n}\right\rangle$ is invariant under the action of the Steenrod algebra. Then they have a non-trivial common zero in $(\mathbb{Z} / 2)^{r}$ if $r>n$.

Proposition $4.7([\mathbf{1 8}])$. Let $f_{1}, \ldots, f_{n}$ be homogeneous polynomials of degree $m$ in $r$ variables with coefficients in $\mathbb{Z} / 2$. Then they have a non-trivial common zero in $(\mathbb{Z} / 2)^{r}$ if $r>m n$.

\section{Induced action on cohomology}

Given a continuous map of topological spaces, determining the induced map on cohomology is a difficult problem in general, even for nice spaces such as spheres. In this section, we show that there are involutions on Milnor manifolds for which the induced action on mod 2 cohomology is non-trivial. First we consider the real case.

Proposition 5.1. Let $A: \mathbb{R} H_{s, s} \longrightarrow \mathbb{R} H_{s, s}$ be the free involution given by

$$
A\left(\left[x_{0}, \ldots, x_{s}\right],\left[y_{0}, \ldots, y_{s}\right]\right) \longmapsto\left(\left[y_{0}, \ldots, y_{s}\right],\left[x_{0}, \ldots, x_{s}\right]\right) .
$$

Then $A^{*}: H^{*}\left(\mathbb{R} H_{s, s}\right) \longrightarrow H^{*}\left(\mathbb{R} H_{s, s}\right)$ is non-trivial.

Proof. By Lemma 2.1,

$$
H^{*}\left(\mathbb{R} H_{s, s} ; \mathbb{Z} / 2\right) \cong \mathbb{Z} / 2[a, b] /\left\langle a^{s+1}, b^{s}+a b^{s-1}+\cdots+a^{s}\right\rangle,
$$

where $a$ and $b$ are homogeneous elements of degree 1 each. By the Künneth formula,

$$
H_{1}\left(\mathbb{R} P^{s} \times \mathbb{R} P^{s}\right) \cong H_{1}\left(\mathbb{R} P^{s}\right) \oplus H_{1}\left(\mathbb{R} P^{s}\right) .
$$

Let $\sigma=\left(\sigma_{1}, \sigma_{2}\right): \Delta^{1} \rightarrow \mathbb{R} P^{s} \times \mathbb{R} P^{s}$ be a singular 1-simplex, and let

$$
A: \mathbb{R} P^{s} \times \mathbb{R} P^{s} \rightarrow \mathbb{R} P^{s} \times \mathbb{R} P^{s}
$$

denote the same involution. Then $A_{*}([\sigma])=[A \circ \sigma]=\left[\left(\sigma_{2}, \sigma_{1}\right)\right]$. This shows that if $a_{1}$ and $b_{1}$ are generators of $H_{1}\left(\mathbb{R} P^{s}\right) \oplus H_{1}\left(\mathbb{R} P^{s}\right)$, then $A_{*}\left(a_{1}\right)=b_{1}$ and hence the induced action on $H_{1}\left(\mathbb{R} P^{s} \times \mathbb{R} P^{s}\right)$ is non-trivial.

Further, we have $H^{1}\left(\mathbb{R} P^{s} \times \mathbb{R} P^{s}\right) \cong H^{1}\left(\mathbb{R} P^{s}\right) \oplus H^{1}\left(\mathbb{R} P^{s}\right)$. If $f \in H^{1}\left(\mathbb{R} P^{s} \times \mathbb{R} P^{s}\right)$, then $A^{*}(f)=f \circ A_{*}$. In particular, $A^{*}(f)\left(a_{1}\right)=f \circ A_{*}\left(a_{1}\right)=f\left(b_{1}\right)$. Choosing $f$ such that $f\left(a_{1}\right) \neq f\left(b_{1}\right)$, we see that $A^{*}$ acts non-trivially on cohomology. Thus, if $a_{2}$ and $b_{2}$ are generators of $H^{1}\left(\mathbb{R} P^{s}\right) \oplus H^{1}\left(\mathbb{R} P^{s}\right)$, then $A^{*}\left(a_{2}\right)=b_{2}$. 
Recall that the real Milnor manifold is also given by the fiber bundle

$$
\mathbb{R} P^{s-1} \stackrel{i}{\hookrightarrow} \mathbb{R} H_{s, s} \stackrel{\pi}{\longrightarrow} \mathbb{R} P^{s} .
$$

Let $\mathbb{R} H_{s, s} \stackrel{j_{1}}{\longrightarrow} \mathbb{R} P^{s} \times \mathbb{R} P^{s}$ be the canonical inclusion, and let

$$
\mathbb{R} P^{s} \stackrel{j_{2}}{\longrightarrow} \mathbb{R} P^{s} \times \mathbb{R} P^{s} \stackrel{p r_{1}}{\longrightarrow} \mathbb{R} P^{s}
$$

be the trivial fiber bundle, where $j_{2}$ is inclusion on to the second factor and $p r_{1}$ is projection on to the first factor. Then we have the following commutative diagram:

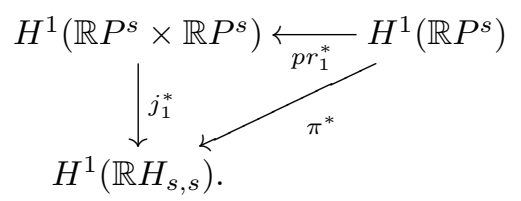

Applying Theorem 4.1 to the fiber bundles given by $\pi$ and $p r_{1}$ and using Theorem 4.2, we get $\pi^{*}\left(a_{2}\right)=a$ and $\operatorname{pr}_{1}^{*}\left(a_{2}\right)=a_{2}$. Further, by commutativity of the diagram, we get $j_{1}^{*}\left(a_{2}\right)=j_{1}^{*}\left(p r_{1}^{*}\left(a_{2}\right)\right)=\pi^{*}\left(a_{2}\right)=a$.

Let $\mathbb{R} P^{s-1} \stackrel{j}{\hookrightarrow} \mathbb{R} P^{s}$ be the canonical inclusion. Then we have the following commutative diagram:

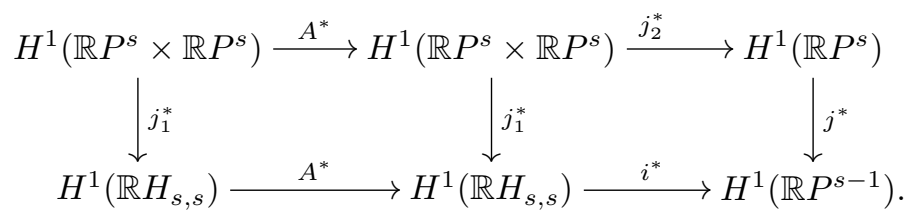

If $b_{3} \in H^{1}\left(\mathbb{R} P^{s-1}\right)$ is the generator, then $j^{*}\left(b_{2}\right)=b_{3}$. Again by Theorems 4.1 and 4.2 , we get $i^{*}(b)=b_{3}$ and $j_{2}^{*}\left(b_{2}\right)=b_{2}$. The commutativity of the right square shows that $j_{1}^{*}\left(b_{2}\right)=b$. The commutativity of the left square gives $A^{*}\left(j_{1}^{*}\left(a_{2}\right)\right)=j_{1}^{*}\left(A^{*}\left(a_{2}\right)\right)$. This implies $A^{*}(a)=j_{1}^{*}\left(b_{2}\right)=b$. Thus the induced map $A^{*}$ is non-trivial.

We have a similar result for the complex case.

Proposition 5.2. Let $B: \mathbb{C} H_{s, s} \longrightarrow \mathbb{C} H_{s, s}$ be the free involution given by

$$
B\left(\left[z_{0}, \ldots, z_{s}\right],\left[w_{0}, \ldots, w_{s}\right]\right) \longmapsto\left(\left[w_{0}, \ldots, w_{s}\right],\left[z_{0}, \ldots, z_{s}\right]\right) .
$$

Then $B^{*}: H^{*}\left(\mathbb{C} H_{s, s}\right) \longrightarrow H^{*}\left(\mathbb{C} H_{s, s}\right)$ is non-trivial.

Proof. The proof is similar to that of the real case and left to the reader.

Note that using propositions 5.1 and 5.2 , we can construct free actions of $(\mathbb{Z} / 2)^{n}$ on $\prod_{i=1}^{n} \mathbb{R} H_{r_{i}, s_{i}}$ and $\prod_{i=1}^{n} \mathbb{C} H_{r_{i}, s_{i}}$ for $1 \leqslant s_{i}=r_{i}$ and $n \geqslant 2$, such that the induced action on mod 2 cohomology is non-trivial.

\section{Proofs of theorems}

\subsection{The real case}

Let $G=(\mathbb{Z} / 2)^{r}$ act freely on a finite-dimensional CW-complex $X \simeq_{2} \prod_{i=1}^{n} \mathbb{R} H_{r_{i}, s_{i}}$ with $1 \leqslant s_{i} \leqslant r_{i}$ for each $1 \leqslant i \leqslant n$. Further, assume that the induced action on mod 
2 cohomology of $X$ is trivial. Using the Künneth formula and Lemma 2.1, we get

$$
H^{*}(X) \cong \mathbb{Z} / 2\left[a_{1}, b_{1}, \ldots, a_{n}, b_{n}\right] / I,
$$

where

$$
\begin{aligned}
I=\left\langle a_{1}^{s_{1}+1}, b_{1}^{r_{1}}+a_{1} b_{1}^{r_{1}-1}+\cdots+a_{1}^{s_{1}} b_{1}^{r_{1}-s_{1}}, \ldots\right. \\
\left.\ldots, a_{n}^{s_{n}+1}, b_{n}^{r_{n}}+a_{n} b_{n}^{r_{n}-1}+\cdots+a_{n}^{s_{n}} b_{n}^{r_{n}-s_{n}}\right\rangle
\end{aligned}
$$

and $a_{1}, b_{1}, \ldots, a_{n}, b_{n}$ are homogeneous elements of degree 1 each. Consider the LeraySerre spectral sequence associated to the Borel fibration

$$
X \hookrightarrow X_{G} \longrightarrow B_{G}
$$

Then we have

$$
E_{2}^{k, l} \cong E_{2}^{k, 0} \otimes E_{2}^{0, l}
$$

where $E_{2}^{k, 0}=H^{k}\left(B_{G}\right)$ and $E_{2}^{0, l}=H^{0}\left(B_{G} ; \mathcal{H}^{l}(X)\right)=H^{l}(X)^{G}=H^{l}(X)$ because the induced action on cohomology is trivial. Thus we have

$$
\begin{aligned}
E_{2}^{*, *} & \cong H^{*}\left(B_{G}\right) \otimes H^{*}(X) \\
& \cong \mathbb{Z} / 2\left[\alpha_{1}, \ldots, \alpha_{r}\right] \otimes \mathbb{Z} / 2\left[a_{1}, b_{1}, \ldots, a_{n}, b_{n}\right] / I .
\end{aligned}
$$

By Theorem 4.3, the spectral sequence does not degenerate at the $E_{2}$ term, and hence $d_{2}: E_{2}^{0,1} \rightarrow E_{2}^{2,0}$ is non-zero. Let $d_{2}\left(1 \otimes a_{i}\right)=u_{i} \otimes 1$ and $d_{2}\left(1 \otimes b_{i}\right)=v_{i} \otimes 1$ for $1 \leqslant i \leqslant n$ with at least one of them being non-zero. Notice that $d_{2}$ is completely determined by $d_{2}\left(1 \otimes a_{i}\right)$ and $d_{2}\left(1 \otimes b_{i}\right)$ as it is a derivation. Consider the ideal

$$
J=\left\langle u_{1}, v_{1}, \ldots, u_{n}, v_{n}\right\rangle
$$

in $H^{*}\left(B_{G}\right)$. Then, using arguments as in [13], we have the following lemma.

Lemma 6.1. Let $G=(\mathbb{Z} / 2)^{r}$ act freely on a finite-dimensional $C W$-complex $X \simeq_{2}$ $\prod_{i=1}^{n} \mathbb{R} H_{r_{i}, s_{i}}$ with $1 \leqslant s_{i} \leqslant r_{i}$ for each $1 \leqslant i \leqslant n$. Further, assume that the induced action on the mod 2 cohomology of $X$ is trivial and that $s_{i}, r_{i} \neq \equiv 3 \bmod 4$ for each $1 \leqslant i \leqslant n$. Then the ideal $J$ in $H^{*}\left(B_{G}\right)$ is invariant under the action of the Steenrod algebra.

Proof. Fix some $1 \leqslant i \leqslant n$. Since $s_{i} \geqslant 1$, we have $a_{i} \neq 0$, and hence $b_{i}^{r_{i}} \neq 0$. Notice that

$$
\begin{aligned}
b_{i}^{r_{i}+1} & =\left(a_{i} b_{i}^{r_{i}-1}+\cdots+a_{i}^{s_{i}} b_{i}^{r_{i}-s_{i}}\right) b_{i} \\
& =a_{i} b_{i}^{r_{i}}+\cdots+a_{i}^{s_{i}} b_{i}^{r_{i}-s_{i}+1} \\
& =a_{i}\left(a_{i} b_{i}^{r_{i}-1}+\cdots+a_{i}^{s_{i}} b_{i}^{r_{i}-s_{i}}\right)+a_{i}^{2} b_{i}^{r_{i}-1}+\cdots+a_{i}^{s_{i}} b_{i}^{r_{i}-s_{i}+1} \\
& =a_{i}^{2} b_{i}^{r_{i}-1}+\cdots+a_{i}^{s_{i}} b_{i}^{r_{i}-s_{i}+1}+a_{i}^{s_{i}+1} b_{i}^{r_{i}-s_{i}}+a_{i}^{2} b_{i}^{r_{i}-1}+\cdots+a_{i}^{s_{i}} b_{i}^{r_{i}-s_{i}+1} \\
& =0 .
\end{aligned}
$$

If $r_{i}$ is even, then

$$
0=d_{2}\left(1 \otimes b_{i}^{r_{i}+1}\right)=\left(1 \otimes b_{i}^{r_{i}}\right) d_{2}\left(1 \otimes b_{i}\right)=v_{i} \otimes b_{i}^{r_{i}} .
$$

Since the map $-\otimes b_{i}^{r_{i}}: E_{2}^{*, 0} \rightarrow E_{2}^{*, r_{i}}$ is injective, we get $v_{i} \otimes 1=0$. Recall that $a_{i}^{s_{i}+1}=0$. Just as above, if $s_{i}$ is even, then $u_{i} \otimes 1=0$. Such $u_{i}$ and $v_{i}$ are obviously invariant under the action of the Steenrod algebra. 
Next let $r_{i}=4 m+1$ and $v_{i} \otimes 1 \neq 0$. Notice that $S q^{1}\left(1 \otimes b_{i}\right)=1 \otimes b_{i}^{2}$ and $d_{2}(1 \otimes$ $\left.b_{i}^{2 m}\right)=0$ by the derivation property of $d_{2}$. Thus $1 \otimes b_{i}^{2 m}$ represents an element in $E_{3}^{0,2 m}$ and we have

$$
0=d_{3}\left(1 \otimes b_{i}^{4 m+2}\right)=\left(1 \otimes b_{i}^{4 m}\right) d_{3}\left(1 \otimes b_{i}^{2}\right) \quad \text { in } \quad E_{3}^{3,4 m} .
$$

Recall that the transgression operator $d_{r}: E_{r}^{0, r-1} \rightarrow E_{r}^{r, 0}$ commutes with the Steenrod operations. In other words, the following diagram commutes:

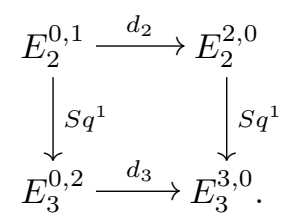

This shows that $d_{3}\left(1 \otimes b_{i}^{2}\right)$ is represented by $S q^{1}\left(v_{i} \otimes 1\right)$. By equation (1) we have that $\left(1 \otimes b_{i}^{4 m}\right) S q^{1}\left(v_{i} \otimes 1\right)$ lies in the image of $d_{2}: E_{2}^{1,4 m+1} \rightarrow E_{2}^{3,4 m}$, and hence

$$
\left(1 \otimes b_{i}^{4 m}\right) S q^{1}\left(v_{i} \otimes 1\right)=\left(1 \otimes b_{i}^{4 m}\right) d_{2}(w)
$$

for some $w \in E_{2}^{1,1}$. Since $1 \otimes b_{i}^{4 m} \neq 0$, the map $-\otimes b_{i}^{4 m}: E_{2}^{*, 0} \rightarrow E_{2}^{*, 4 m}$ is injective, we get $S q^{1}\left(v_{i} \otimes 1\right)=d_{2}(w)$. Let

$$
w=\sum_{j, k} \lambda_{j, k}\left(\alpha_{j} \otimes a_{k}\right)+\sum_{j, k} \mu_{j, k}\left(\alpha_{j} \otimes b_{k}\right)
$$

where $\lambda_{j, k}, \mu_{j, k} \in \mathbb{Z} / 2$. Then

$$
d_{2}(w)=\sum_{j, k} \lambda_{j, k}\left(\alpha_{j} u_{k} \otimes 1\right)+\sum_{j, k} \mu_{j, k}\left(\alpha_{j} v_{k} \otimes 1\right) \in J .
$$

This shows that $S q^{1}\left(v_{i} \otimes 1\right) \in J$. Similarly, if $s_{i}=4 m+1$ and $u_{i} \otimes 1 \neq 0$, then $S q^{1}\left(u_{i} \otimes 1\right) \in J$. Hence $J$ is invariant under the action of the Steenrod algebra.

For an integer $n$, define

$$
\eta(n)= \begin{cases}0 & \text { if } n \text { is even } \\ 1 & \text { if } n \text { is odd }\end{cases}
$$

\section{Proof of Theorem 1.1}

We first prove (1). As noticed in the proof of Lemma 6.1, if some $s_{i}, r_{i}$ is even, then the corresponding $u_{i}, v_{i}$ is zero. Regard $u_{1}, v_{1}, \ldots, u_{n}, v_{n} \in H^{2}\left(B_{G}\right)$ as homogeneous polynomials of degree two in $r$ variables. Suppose that they have a non-trivial common zero in $(\mathbb{Z} / 2)^{r}$. Then by Proposition 4.5 , there is a subgroup inclusion

$$
j: \mathbb{Z} / 2 \hookrightarrow(\mathbb{Z} / 2)^{r}
$$

such that $j^{*}\left(u_{i}\right)=0=j^{*}\left(v_{i}\right)$ for each $1 \leqslant i \leqslant n$. Restrict the $G$ action on $X$ to $\mathbb{Z} / 2$ action on $X$, and consider the Leray-Serre spectral sequence $\left\{\bar{E}_{r}^{*, *}, \bar{d}_{r}\right\}$ associated to 
the Borel fibration

$$
X \hookrightarrow X_{\mathbb{Z} / 2} \longrightarrow B_{\mathbb{Z} / 2} .
$$

The naturality of the Leray-Serre spectral sequence gives the following commutative diagram:

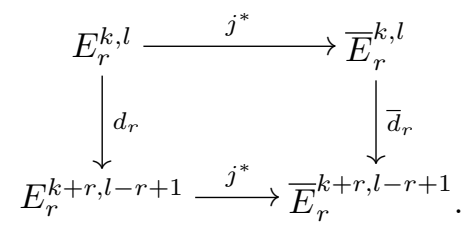

Observe that $j^{*}: E_{2}^{0, l} \rightarrow \bar{E}_{2}^{0, l}$ is the identity map. This, together with the commutative diagram, gives $\bar{d}_{2}\left(1 \otimes a_{i}\right)=0=\bar{d}_{2}\left(1 \otimes b_{i}\right)$ for each $1 \leqslant i \leqslant n$. Hence $\bar{d}_{r}=0$ for each $r \geqslant 2$ and

$$
\bar{E}_{2}^{*, *}=\bar{E}_{\infty}^{*, *} .
$$

But we have $H^{*}\left(X_{G}\right) \cong \operatorname{Tot} E_{\infty}^{*, *}$. This implies that $H^{*}\left(X_{G}\right)$ is infinite dimensional, a contradiction by Proposition 4.4. Hence the system of homogeneous polynomials does not have any non-trivial common zero in $(\mathbb{Z} / 2)^{r}$. Thus, by Proposition 4.7, $r \leqslant 2\left(\eta\left(s_{1}\right)+\eta\left(r_{1}\right)+\cdots+\eta\left(s_{n}\right)+\eta\left(r_{n}\right)\right)$.

Next, we prove (2). By Lemma 6.1, the ideal $J$ is invariant under the action of the Steenrod algebra. By the discussion above, the system of homogeneous polynomials $u_{1}, v_{1}, \ldots, u_{n}, v_{n}$ do not have any non-trivial common zero in $(\mathbb{Z} / 2)^{r}$. Thus, by Proposition 4.6, we have $r \leqslant\left(\eta\left(s_{1}\right)+\eta\left(r_{1}\right)+\cdots+\eta\left(s_{n}\right)+\eta\left(r_{n}\right)\right)$. This completes the proof of Theorem 1.1.

\subsection{The complex case}

Let $G=(\mathbb{Z} / 2)^{r}$ act freely on a finite-dimensional CW-complex $X \simeq_{2} \prod_{i=1}^{n} \mathbb{C} H_{r_{i}, s_{i}}$ with $1 \leqslant s_{i} \leqslant r_{i}$ for each $1 \leqslant i \leqslant n$. And suppose that the induced action on mod 2 cohomology of $X$ is trivial. Using Lemma 2.1, we get

$$
H^{*}(X) \cong \mathbb{Z} / 2\left[g_{1}, h_{1}, \ldots, g_{n}, h_{n}\right] / K,
$$

where $K$ is the ideal

$$
\left\langle g_{1}^{s_{1}+1}, h_{1}^{r_{1}}+g_{1} h_{1}^{r_{1}-1}+\cdots+g_{1}^{s_{1}} h_{1}^{r_{1}-s_{1}}, \ldots, g_{n}^{s_{n}+1}, h_{n}^{r_{n}}+g_{n} h_{n}^{r_{n}-1}+\cdots+g_{n}^{s_{n}} h_{n}^{r_{n}-s_{n}}\right\rangle
$$

and $g_{1}, h_{1}, \ldots, g_{n}, h_{n}$ are all homogeneous elements of degree 2 each. As in the real case, we have

$$
E_{2}^{* * *} \cong \mathbb{Z} / 2\left[\alpha_{1}, \ldots, \alpha_{r}\right] \otimes \mathbb{Z} / 2\left[g_{1}, h_{1}, \ldots, g_{n}, h_{n}\right] / K .
$$

Again by Theorem 4.3, the spectral sequence does not degenerate at the $E_{2}$ term. Notice that $d_{r}=0$ for all even $r$. In particular, $d_{2}=0$, and hence $d_{3}: E_{3}^{0,2} \rightarrow E_{3}^{3,0}$ must be non-zero. Let $d_{3}\left(1 \otimes g_{i}\right)=x_{i} \otimes 1$ and $d_{3}\left(1 \otimes h_{i}\right)=y_{i} \otimes 1$ for $1 \leqslant i \leqslant n$ with at least one of them being non-zero. Consider the ideal

$$
L=\left\langle x_{1}, y_{1}, \ldots, x_{n}, y_{n}\right\rangle
$$

in $H^{*}\left(B_{G}\right)$. Then we have the following lemma. 
Lemma 6.2. Let $G=(\mathbb{Z} / 2)^{r}$ act freely on a finite-dimensional $C W$-complex $X \simeq_{2}$ $\prod_{i=1}^{n} \mathbb{C} H_{r_{i}, s_{i}}$ with $1 \leqslant s_{i} \leqslant r_{i}$ for each $1 \leqslant i \leqslant n$. Suppose that the induced action on mod 2 cohomology of $X$ is trivial and $s_{i}, r_{i} \not \equiv 3 \bmod 4$ for each $1 \leqslant i \leqslant n$. Then the ideal $L$ in $H^{*}\left(B_{G}\right)$ is invariant under the action of the Steenrod algebra.

Proof. We describe the proof briefly as it is similar to the proof of Lemma 6.1. Fix some $1 \leqslant i \leqslant n$. Since $s_{i} \geqslant 1$, we have $h_{i}^{r_{i}} \neq 0$. Notice that $h_{i}^{r_{i}+1}=0$. If $r_{i}$ is even, then

$$
0=d_{3}\left(1 \otimes h_{i}^{r_{i}+1}\right)=y_{i} \otimes h_{i}^{r_{i}}
$$

Since the map $-\otimes h_{i}^{r_{i}}: E_{3}^{*, 0} \rightarrow E_{3}^{*, r_{i}}$ is injective, we get $y_{i} \otimes 1=0$. Since $g_{i}^{s_{i}+1}=0$, it follows that if $s_{i}$ is even, then $x_{i} \otimes 1=0$. Such $x_{i}$ and $y_{i}$ are obviously invariant under the action of the Steenrod algebra.

Let $r_{i}=4 m+1$ and $y_{i} \otimes 1 \neq 0$. Notice that $S q^{1}\left(1 \otimes h_{i}\right)=0$ and the following diagram commutes:

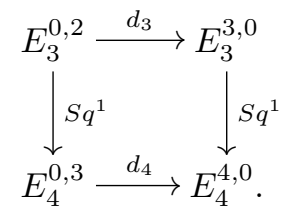

Since $d_{4}=0$, the commutativity of the diagram shows that $0=S q^{1}\left(y_{i} \otimes 1\right) \in L$.

Next we have $S q^{2}\left(1 \otimes h_{i}\right)=1 \otimes h_{i}^{2}$. Further, $d_{3}\left(1 \otimes h_{i}^{2 m}\right)=0$, and hence $1 \otimes h_{i}^{2 m}$ represents an element in $E_{5}^{0,4 m}$. Since $h_{i}^{4 m+2}=0$, we have

$$
0=d_{5}\left(1 \otimes h_{i}^{4 m+2}\right)=\left(1 \otimes h_{i}^{4 m}\right) d_{5}\left(1 \otimes h_{i}^{2}\right) \quad \text { in } \quad E_{5}^{5,4 m} .
$$

Consider the following commutative diagram:

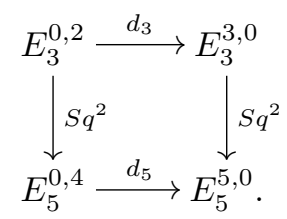

This shows that $d_{5}\left(1 \otimes h_{i}^{2}\right)$ is represented by $S q^{2}\left(y_{i} \otimes 1\right)$. By equation (3) we have that $\left(1 \otimes h_{i}^{4 m}\right) S q^{2}\left(y_{i} \otimes 1\right)$ lies in the image of $d_{3}: E_{3}^{2,4 m+2} \rightarrow E_{3}^{5,4 m}$ and hence

$$
\left(1 \otimes h_{i}^{4 m}\right) S q^{2}\left(y_{i} \otimes 1\right)=\left(1 \otimes h_{i}^{4 m}\right) d_{3}(z)
$$

for some $z \in E_{3}^{2,2}$. Since $1 \otimes h_{i}^{4 m} \neq 0$, the map $-\otimes h_{i}^{4 m}: E_{3}^{*, 0} \rightarrow E_{3}^{*, 4 m}$ is injective, and we get $S q^{2}\left(y_{i} \otimes 1\right)=d_{3}(z)$. Let

$$
z=\sum_{j, k, l} \lambda_{j, k, l}\left(\alpha_{j} \alpha_{k} \otimes g_{l}\right)+\sum_{j, k, l} \mu_{j, k, l}\left(\alpha_{j} \alpha_{k} \otimes h_{l}\right),
$$

where $\lambda_{j, k, l}, \mu_{j, k, l} \in \mathbb{Z} / 2$. Then

$$
d_{3}(z)=\sum_{j, k, l} \lambda_{j, k, l}\left(\alpha_{j} \alpha_{k} x_{l} \otimes 1\right)+\sum_{j, k, l} \mu_{j, k, l}\left(\alpha_{j} \alpha_{k} y_{l} \otimes 1\right) \in L .
$$

This shows that $S q^{2}\left(y_{i} \otimes 1\right) \in L$. Similarly, if $s_{i}=4 m+1$ and $x_{i} \otimes 1 \neq 0$, then 
$S q^{1}\left(x_{i} \otimes 1\right), S q^{2}\left(x_{i} \otimes 1\right) \in L$. Hence $L$ is invariant under the action of the Steenrod algebra.

\section{Proof of Theorem 1.2}

If some $s_{i}, r_{i}$ is even, then the corresponding $x_{i}, y_{i}$ is zero. Suppose $x_{1}, y_{1}, \ldots, x_{n}, y_{n}$ have a non-trivial common zero in $(\mathbb{Z} / 2)^{r}$. Then by Proposition 4.5 there is a subgroup inclusion

$$
j: \mathbb{Z} / 2 \hookrightarrow(\mathbb{Z} / 2)^{r}
$$

such that $j^{*}\left(x_{i}\right)=0=j^{*}\left(y_{i}\right)$ for each $1 \leqslant i \leqslant n$. Restrict the $G$ action on $X$ to $\mathbb{Z} / 2$ action on $X$, and consider the Leray-Serre spectral sequence $\left\{\bar{E}_{r}^{*, *}, \bar{d}_{r}\right\}$ associated to the Borel fibration

$$
X \hookrightarrow X_{\mathbb{Z} / 2} \rightarrow B_{\mathbb{Z} / 2}
$$

Observe that $j^{*}: E_{3}^{0, l} \rightarrow \bar{E}_{3}^{0, l}$ is the identity map. This, together with the commutative diagram (2), gives $\bar{d}_{3}\left(1 \otimes g_{i}\right)=0=\bar{d}_{3}\left(1 \otimes h_{i}\right)$ for each $1 \leqslant i \leqslant n$. Hence $\bar{d}_{r}=0$ for each $r \geqslant 2$ and $\bar{E}_{2}^{*, *}=\bar{E}_{\infty}^{* * *}$. This gives a contradiction by Proposition 4.4. Hence the system of homogeneous polynomials does not have any non-trivial common zero in $(\mathbb{Z} / 2)^{r}$. Thus, by Proposition $4.7, r \leqslant 3\left(\eta\left(s_{1}\right)+\eta\left(r_{1}\right)+\cdots+\eta\left(s_{n}\right)+\eta\left(r_{n}\right)\right)$. This proves (1).

By Lemma 6.2, the ideal $L$ is invariant under the action of the Steenrod algebra. By the above discussion, the system of homogeneous polynomials $x_{1}, y_{1}, \ldots, x_{n}, y_{n}$ does not have any non-trivial common zero in $(\mathbb{Z} / 2)^{r}$. Thus, by Proposition 4.6 , we have $r \leqslant\left(\eta\left(s_{1}\right)+\eta\left(r_{1}\right)+\cdots+\eta\left(s_{n}\right)+\eta\left(r_{n}\right)\right)$. This proves Theorem 1.2(2).

Restricting to actions of elementary abelian 2-groups on $\prod_{i=1}^{n} \mathbb{C} H_{r_{i}, s_{i}}$ for which the induced action on mod 2 cohomology is trivial, we obtain the following corollary.

Corollary 6.3. Let $1 \leqslant s_{i}<r_{i}$ for each $1 \leqslant i \leqslant n$. Then

$$
\operatorname{frk}_{2}\left(\prod_{i=1}^{n} \mathbb{C} H_{r_{i}, s_{i}}\right)=\eta\left(s_{1}\right)+\eta\left(r_{1}\right)+\cdots+\eta\left(s_{n}\right)+\eta\left(r_{n}\right)
$$

whenever $s_{i} \equiv 1 \bmod 4$ and $r_{i} \equiv 0,2 \bmod 4$.

Proof. Theorem 1.2(2) gives the upper bound. In Section 3, we constructed a $\mathbb{Z} / 2$ action on $\mathbb{C} H_{r_{i}, s_{i}}$ when $s_{i}$ is odd and $r_{i}$ is even, for which the induced action on $H^{*}\left(\mathbb{C} H_{r_{i}, s_{i}}\right)$ is trivial. The products of these actions on $\prod_{i=1}^{n} \mathbb{C} H_{r_{i}, s_{i}}$ achieve the desired bound when $s_{i} \equiv 1 \bmod 4$ and $r_{i} \equiv 0,2 \bmod 4$.

\section{Some concluding remarks}

We conclude with the following remarks on our results. Adem and Yalçın asked the following question $[\mathbf{3}, \mathrm{p} .70]$.

Question. If $(\mathbb{Z} / 2)^{r}$ acts freely on a finite $C W$-complex $X$ with mod 2 cohomology generated by one-dimensional classes, does it follows that $r \leqslant 2 \operatorname{dim} H_{1}(X ; \mathbb{Z} / 2)$ ? 
Let $(\mathbb{Z} / 2)^{r}$ act freely on a finite $\mathrm{CW}$-complex $X \simeq_{2} \prod_{i=1}^{n} \mathbb{R} H_{r_{i}, s_{i}}$ such that the induced action on mod 2 cohomology is trivial. If $1 \leqslant s_{i}<r_{i}$ for each $1 \leqslant i \leqslant n$, then

$$
H_{1}\left(\mathbb{R} H_{r_{i}, s_{i}} ; \mathbb{Z} / 2\right)=\mathbb{Z} / 2 \oplus \mathbb{Z} / 2,
$$

and hence $\operatorname{dim} H_{1}(X ; \mathbb{Z} / 2)=2 n$. Therefore, by Theorem 1.1(1),

$$
r \leqslant 2\left(\eta\left(s_{1}\right)+\eta\left(r_{1}\right)+\cdots+\eta\left(s_{n}\right)+\eta\left(r_{n}\right)\right) \leqslant 2(2 n)=2 \operatorname{dim} H_{1}(X ; \mathbb{Z} / 2) .
$$

Thus the above question has a positive answer for $X \simeq_{2} \prod_{i=1}^{n} \mathbb{R} H_{r_{i}, s_{i}}$.

Let $X$ be as in Theorem 1.1 and Theorem 1.2. Let $s_{i}$ be even and $r_{i}$ be odd for each $1 \leqslant i \leqslant n$. If $X \simeq_{2} \prod_{i=1}^{n} \mathbb{R} H_{r_{i}, s_{i}}$, then the Euler characteristic $\chi(X)=1$. Similarly, if $X \simeq_{2} \prod_{i=1}^{n} \mathbb{C} H_{r_{i}, s_{i}}$, then $\chi(X)$ is odd. Hence no elementary abelian 2-group can act freely on $X$ and our theorems are weak in this case.

It is well known that if a closed smooth manifold does not bound mod 2, then it does not admit any free involution. It was shown in $[\mathbf{2 2}]$ that, $\mathbb{R} H_{r, s}$ does not bound for $s=2 k+1$ and $r=2^{\beta}(2 l+1)$ if and only if one of the following holds:

- $\beta \geqslant 2$ and $k \geqslant 1$,

- $\beta=1, l+1=2^{\delta}(2 t+1)$ and $k \geqslant 2^{\delta+1}-1$.

Thus $\mathbb{R} H_{r, s}$ does not admit any free involution in these cases.

\section{Acknowledgments}

A part of this work was done when the author was visiting the International Centre for Theoretical Physics (ICTP) in Trieste during March to May of 2013. The author is grateful to the ICTP for providing financial support and excellent working atmosphere. The author also thanks the Department of Science and Technology of India for support via the INSPIRE Faculty Scheme IFA-11MA-01/2011 and the SERC Fast Track Scheme SR/FTP/MS-027/2010.

\section{References}

[1] A. Adem \& W. Browder, The free rank of symmetry of $\left(\mathbb{S}^{n}\right)^{k}$, Invent. Math. 92 (1988), 431-440.

[2] A. Adem \& J.F. Davis, Topics in transformation groups, Handbook of Geometric Topology, 1-54, North-Holland, Amsterdam, 2002.

[3] A. Adem \& E. Yalçın, On some examples of group actions and group extensions, J. Group Theory 2 (1999), 69-79.

[4] C. Allday \& V. Puppe, Cohomological methods in transformation groups, Cambridge Studies in Advanced Mathematics 32, Cambridge University Press, Cambridge, 1993.

[5] C. Allday, Elementary abelian p-group actions on lens spaces, Topology Hawaii (Honolulu, HI, 1990), 1-11, World Sci. Publishing, River Edge, NJ, 1992.

[6] D.J. Benson \& J.F. Carlson, Complexity and multiple complexes, Math. Z. 195 (1987), 221-238.

[7] A. Borel et al., Seminar on transformation groups, Annals of Mathematics Studies 46, Princeton University Press, 1960. 
[8] V.M. Bukhshtaber \& N. Ray, Toric manifolds and complex cobordisms, Russian Math. Surveys 53 (1998), 371-373.

[9] G. Carlsson, On the non-existence of free actions of elementary abelian groups on products of spheres, Amer. J. Math. 102 (1980), 1147-1157.

[10] G. Carlsson, On the rank of abelian groups acting freely on $\left(\mathbb{S}^{n}\right)^{k}$, Invent. Math. 69 (1982), 393-400.

[11] P.E. Conner, On the action of a finite group on $\mathbb{S}^{n} \times \mathbb{S}^{n}$, Ann. of Math. 66 (1957), 586-588.

[12] P.E. Conner \& E.E. Floyd, Differentiable periodic maps, Ergebn. Math. 33, Springer, 1964.

[13] L.W. Cusick, Elementary abelian 2-groups that act freely on products of real projective spaces, Proc. Amer. Math. Soc. 87 (1983), 728-730.

[14] L.W. Cusick, Finite groups that can act freely on products of even-dimensional spheres, Indiana Univ. Math. J. 35 (1986), 175-178.

[15] L.W. Cusick, Free actions on products of even-dimensional spheres, Proc. Amer. Math. Soc. 99 (1987), 573-574.

[16] L.W. Cusick, Free actions on spaces with nonzero Euler characteristic, Topology Appl. 33 (1989), 185-196.

[17] I. Gálvez \& A. Tonks, Differential operators and the Witten genus for projective spaces and Milnor manifolds, Math. Proc. Cambridge Philos. Soc. 135 (2003), $123-131$.

[18] M. Greenberg, Lectures on Forms in Many Variables, Benjamin, New York, 1969.

[19] B. Hanke, The stable free rank of symmetry of products of spheres, Invent. Math. 178 (2009), 265-298.

[20] A. Heller, A note on spaces with operators, Illinois J. Math. 3 (1959), 98-100.

[21] M. Kamata \& K. Ono, On the multiple points of the self-transverse immersions of the real projective space and the Milnor manifold, Kyushu J. Math. 60 (2006), 331-344.

[22] S.S. Khare \& A.K. Das, Which Milnor manifolds bound?, Indian J. Pure Appl. Math. 31 (2000), 1503-1513.

[23] R.E. Mosher \& M.C. Tangora, Cohomology operations and applications in homotopy theory, Harper \& Row, New York/London, 1968.

[24] J. McCleary, A user's guide to spectral sequences, Cambridge Studies in Advanced Mathematics 58, Second Edition, Cambridge University Press, Cambridge, 2001.

[25] J.W. Milnor, On the Stiefel-Whitney numbers of complex manifolds and spin manifolds, Topology 3 (1965), 223-230.

[26] H.K. Mukerjee, Classification of homotopy real Milnor manifolds, Topology Appl. 139 (2004), 151-184.

[27] O.B. Okutan \& E. Yalçın, Free actions on products of spheres at high dimensions, Algebr. Geom. Topol. 13 (2013), 2087-2099. 
[28] R. Oliver, Free compact group actions on products of spheres, Algebraic Topology, Aarhus, 1978 (Proc. Sympos., Univ. Aarhus, Aarhus, 1978), 539-548, Lecture Notes in Mathematics 763, Springer, Berlin, 1979.

[29] P.A. Smith, Permutable periodic transformations, Proc. Nat. Acad. Sci. USA. 30 (1944), 105-108.

[30] R.G. Swan, Periodic resolutions for finite groups, Ann. of Math. 94 (1960), 267-291.

[31] E. Yalçın, Free actions of p-groups on products of lens spaces, Proc. Amer. Math. Soc. 129 (2001), 887-898.

[32] E. Yalçın, Group actions and group extensions, Trans. Amer. Math. Soc. 352 (2000), 2689-2700.

Mahender Singh mahender@iisermohali.ac.in

Indian Institute of Science Education and Research (IISER) Mohali, Sector 81, Knowledge City, S A S Nagar (Mohali), Post Office Manauli, Punjab 140306, India. 